\title{
Fertility and reproductive outcome after tubal ectopic pregnancy: comparison among methotrexate, surgery and expectant management
}

\author{
Silvia Baggio ${ }^{1,2}$ (D) Simone Garzon ${ }^{3} \cdot$ Anna Russo $^{1} \cdot$ Cesare Quintino lanniciello ${ }^{1}$. Lorenza Santi ${ }^{4}$. \\ Antonio Simone Laganà ${ }^{3} \cdot$ Ricciarda Raffaelli $^{1} \cdot$ Massimo Franchi ${ }^{1}$
}

Received: 4 April 2020 / Accepted: 13 August 2020 / Published online: 27 August 2020

(C) The Author(s) 2020

\begin{abstract}
Purpose To compare fertility and reproductive outcome after surgical, medical, and expectant management for tubal ectopic pregnancy (EP).

Methods 133 of 228 patients, who were managed between January 2012 and December 2017 for a tubal EP, tried to conceive immediately after treatment: 86 out of 173 (49.7\%) underwent surgical treatment; 38 (21.9\%) were treated with methotrexate (MTX), and 49 (28.3\%) had expectant management. Clinical data were retrieved by medical records, fertility outcomes were obtained by phone follow-up. The cumulative incidence (CI) of intrauterine clinical pregnancy (CP), miscarriage, live birth (LB), and recurrent EP, and the time between treatment and first intrauterine $\mathrm{CP}$ were compared between women treated with MTX, surgery and expectant management.

Results The CI of intrauterine CP starting from 12 months after the EP was $65.3 \%$ for the expectant management, 55.3\% for the MTX group, and $39.5 \%$ for surgery $(p=0.012)$. Post-hoc analysis showed expectant management having higher intrauterine CP and LB, and shorter time between treatment and first intrauterine CP compared to surgery $(p<0.05)$. The $\mathrm{CI}$ of recurrent EP was comparable between the 3 groups. The analysis stratified per $\beta$ hCG cut-off of $1745 \mathrm{mUI} / \mathrm{mL}$ and EP mass cut-off of $25 \mathrm{~mm}$ reported consistent results.

Conclusions Women successfully managed by expectation appear to have better reproductive outcomes compared to women who underwent surgery, with the shortest time to achieve a subsequent intrauterine CP. Therefore, if safely applicable the expectant management should be considered in the case of tubal EP. The fact that the chosen treatment was primarily guided by the $\beta \mathrm{hCG}$ value and EP mass diameter based on the protocol, which is intrinsically related to the characteristics of the EP, represents the main limitation of the present study. Indeed, we cannot completely exclude that the observed differences between treatments are related to the EP itself instead of the treatment.
\end{abstract}

Keywords Ectopic pregnancy $\cdot$ Methotrexate $\cdot$ Fertility $\cdot$ Salpingectomy $\cdot$ Pregnancy outcome

\section{Introduction}

Silvia Baggio

silvia.baggio1@gmail.com

1 Department of Obstetrics and Gynecology, AOUI Verona, University of Verona, Verona, Italy

2 Department of Obstetrics and Gynecology, "Sacro Cuore" Hospital, Negrar Di Valpolicella, Verona, Italy

3 Department of Obstetrics and Gynecology, "Filippo Del Ponte" Hospital, University of Insubria, Varese, Italy

4 Department of Endocrinology, Diabetes and Metabolism, AOUI Verona, University of Verona, Verona, Italy
Ectopic pregnancy (EP) occurs when the fertilized ovum implants outside the endometrial cavity with an incidence of $1 \%$ of all pregnancies [1]. Tubal localization accounts for $95-99 \%$ of ectopic pregnancies. Other localizations such as ovarian, cervical, cornual, and abdominal implants are rarely seen $[2,3]$. In the past decades, the management of EP was revolutionized by the development and continuous improvement of transvaginal ultrasonography (TVUS), which, together with the implementation of the Beta human chorionic gonadotropin ( $\beta \mathrm{hCG}$ ) assay, allows early diagnosis of EP with the prevention of complications [4]. As a 
consequence, the clinical presentation of EP has changed from a life-threatening disease, necessitating emergency surgery, to a benign condition in almost asymptomatic women for whom non-surgical treatment options are also available [5].

With the development of laparoscopic techniques, rapid and mini-invasive intervention is quite always possible [6], which may be radical, by the removal of the entire fallopian tube (salpingectomy), or conservative, with the only removal of the products of gestation from the tube by salpingotomy or tubal milking [7,8]. Non-surgical treatments of EP include expectant management and intramuscular or intravenous Methotrexate (MTX) injection, which avoid the potential complications of surgery. Treatment of uncomplicated EPs with MTX was reported effective, safe, and less costly as compared to surgery [5, 9].

The best approach is tailored to the patients' medical status, success rate, complications rate, side effects, and costs. Nevertheless, even fertility outcomes after the episode are important variables to consider in choosing the treatment modality [10]. However, it remains unclear which treatment is the best regarding subsequent fertility [11], in particular, there are still insufficient data about treatment success and future fertility rates in EP cases managed expectantly [12-15]. On that bases, we performed a cohort study aimed to compare the success rate and the impact on fertility and reproductive outcomes of the surgical, medical, and expectant management for tubal EP.

\section{Materials and methods}

\section{Study population}

Women diagnosed with an EP between January 2012 and December 2017 in the department of Obstetrics and Gynecology of the "Ospedale Donna Bambino" in Verona (Italy) were identified searching in the records of all hospitalization performed during that period, using the diagnosis-related group (DRG) of EP at admission and/or at discharge. We also performed a crossmatch search among Gynecological Department DRG data and the Pharmacological Department Registers, to identify all gynecological patients who received an injection of MTX. Moreover, a crossmatch search was performed with the Gynecological Surgical register to identify the records of all surgeries performed for EP. The medical records of all identified cases were retrieved, and the data were extracted.

Patients who were admitted for the first episode of clinically suspected tubal EP were eligible. When one patient had more than one EP during the study period, only the first episode was considered. If the patient had the first episode out of the study period or at another center, the woman was excluded from the study. Patients with no tubal EP (cervical, ovarian, cornual, subhepatic, interstitial implantation) or EP with unknown localization were excluded. Clinically suspected EP was defined by the presence in the medical records of two criteria: direct transvaginal ultrasound (US) showing specific signs, such as hematosalpinx or lateral uterine gestational sac and empty uterus; and positive $\beta \mathrm{hCG}$ having suggestive kinetics, such as no doubling, little reduction or stability of $\beta \mathrm{hCG}$ levels after $48 \mathrm{~h}$. We considered eligible only patients with an empty uterus and a sonographic suspect of tubal EP mass, in order to exclude early spontaneous miscarriages. The dimension of the gestational sac was retrieved by medical records; if a gestational sac was not reported, the patient was excluded from the current analysis. The presence or absence of clinical signs or symptoms, such as pelvic pain and metrorrhagia, were not required.

For all eligible patients, a phone follow-up was performed after 12-80 months from treatment, only one telephone call for each patient was done. Women reporting no desire of subsequent pregnancy after the index EP, and who, therefore, did not actively try to conceive were excluded. Patients who actively tried to conceive after the index EP and who accepted to be involved in the study were included. After consent and inclusion, a telephone interview was submitted with questions focused on fertility outcomes after the index EP. The questions were aimed to investigate the diagnosis of intrauterine clinical pregnancy $(\mathrm{CP})$, miscarriages (pregnancy loss before 24 gestational weeks), live births (LBs) (delivery after 24 gestational weeks), recurrent EP, mode of conception, and details about any subsequent surgery after the index EP. The time between the treatment of the index $\mathrm{EP}$ and each recorded outcome was estimated from the date of the index EP treatment (or discharge in case of expectant management) until the last period date before the $\mathrm{CP}$ or recurrence EP.

Demographic characteristics of included patients such as age, smoking status, body mass index (BMI), gravid, parity before index EP (miscarriage, abortion, previous EP, live births), blood group, and Rh status were retrieved from medical records. From medical records, data regarding the access at the emergency department on the day of diagnosis (cause of the access, symptoms, levels of $\beta \mathrm{hCG}$, hemoglobin, platelets, white cells, AST, ALT, Creatinine, and US main diameter of the mass indicated in the report) were also retrieved. All data were collected in a database.

\section{Management}

According to the protocol adopted at the "Ospedale Donna e Bambino" and consistent with the literature (Table 1) [4, 11], after sonographic, biochemical, and clinical evaluation, the gynecologist decided the treatment of the clinically suspected tubal EP. Those who did not require an 
Table 1 Management protocol of ectopic pregnancy applied to the study population

\begin{tabular}{lllll}
\hline Admission & EP mass diameter & \multicolumn{4}{l}{ Evaluation of $\beta \mathrm{hCG}$ after $48 \mathrm{~h}$} & \\
\cline { 3 - 5 } $\mathrm{mUI} / \mathrm{mL}$ & & $\uparrow$ & $\approx$ & $\downarrow$ \\
\hline$\beta \mathrm{hCG} \leq 1000$ & $<35 \mathrm{~mm}$ & MTX & Expectant/MTX & Expectant/MTX \\
& Absent & Expectant & Expectant & Expectant \\
$1000<\beta \mathrm{hCG} \leq 2000$ & $<35 \mathrm{~mm}$ & Surgery & Surgery/MTX & MTX/expectant \\
& Absent & Monitor- & Monitor- & Expectant/MTX \\
& & ing + curet- & ing + curettage/ & \\
& & tage/MTX & (MTX) & \\
$\beta \mathrm{hCG}>2000$ & $<35 \mathrm{~mm}$ & Surgery & Surgery & Surgery/MTX \\
& Absent & Surgery/MTX & Surgery/MTX & Expectant + curettage/MTX \\
& & &
\end{tabular}

$E P$ ectopic mass, $\beta h C G$ beta-human chorionic gonadotropin, $M T X$ methotrexate immediate surgical intervention underwent repeated blood tests for $\beta$ hCG levels and complete blood cell count, as well as repeated US examinations and measurements of blood pressure and pulse, in order to decide the best treatment, according to the protocol.

In the case of expectant management or medical treatment, women were discharged and followed up in the outpatient regimen. They must be asymptomatic during the hospitalization and they had to check $\beta$ hCG values every 7 days until negative values $(<5 \mathrm{UI} / \mathrm{L})$. In the case of pain or rising of the $\beta \mathrm{hCG}$ levels, the management option was reconsidered.

Patients were eligible for medical treatment consistently with protocol showed in Table 1 and if they satisfied the following conditions: (a) no intrauterine pregnancy sac on US (synchronous orthotopic pregnancy); (b) hemodynamic stability; (c) normal results of the liver and renal function tests; (d) patient's consent; and (e) no known allergy to MTX. Medical treatment consisted of a single dose of intramuscular injection of MTX $\left(50 \mathrm{mg} / \mathrm{m}^{2}\right)$, without the alternating administration of folinic acid [16]. The protocol required that the $\beta \mathrm{hCG}$ levels were measured on days 0,4 , and 7 after MTX. The success of medical treatment was defined as the drop of $\beta$ hCG levels between days 4 and 7 , which allowed for weekly biological follow up till resolution $(\beta \mathrm{hCG}<5 \mathrm{UI} / \mathrm{L})$. Conversely, a reiteration of medical management with reinjection of MTX $\left(50 \mathrm{mg} / \mathrm{m}^{2}\right)$ was required when there was a growth of $\beta \mathrm{hCG}$ levels between days 4 and 7 or if during serial biological monitoring $\beta$ hCG value remained $\geq 5 \mathrm{UI} / \mathrm{L}$. Failure of medical treatment was considered when the growth of $\beta$ hCG levels was observed despite the repetition of MTX injection or patient clinical worsening after the first or second MTX injection. Those receiving MTX were informed about the possible side effects and the interactions with alcohol, non-steroidal anti-inflammatory drugs, aspirin, and antibiotics, and received advice on fluid intake, buccal hygiene, and exposure to sunlight. They were advised to use adequate contraception for 3 months after the last injection of MTX [17].
In no symptomatic women with EP mass $<35 \mathrm{~mm}$ and free fluid in Douglas lower than $100 \mathrm{~mL}$, both expectant management and MTX treatment were possible. The decision between the two managements was made considering mainly $\beta$ hCG levels and its dynamic after $48 \mathrm{~h}$. Unless rapid increase of $\beta \mathrm{hCG}$, expectant management was preferred for values $<1000 \mathrm{mUI} / \mathrm{mL}$ or in case of decreasing trend; for values stable between 1000 and $2000 \mathrm{mUI} / \mathrm{mL}$, both expectant and MTX were considered, although MTX management was usually preferred (Table 1).

The surgical approach was offered to patients with severe symptoms or signs suggesting surgical complications (intraabdominal bleeding $>100 \mathrm{~mL}$, acute abdomen, shock, presence of the extrauterine fetal heartbeat, diameter of the mass $>35 \mathrm{~mm}$ ), these patients were immediately treated surgically. Moreover, surgical treatment was performed in case of high stable or increasing $\beta \mathrm{hCG}$ values or if previous treatments failed. There were three options for surgical treatment: tubal or fimbriae milking, salpingotomy, and salpingectomy. Salpingectomy was the treatment of choice at our Institution, due to the reported lower rate of recurrence with no significant difference in future fertility compared to salpingotomy $[7,18,19]$. To evaluate fertility outcomes, we considered only the final treatment.

\section{Statistical analysis}

Statistical analysis was performed using SPSS for Windows V.21.0 (IBM Corporation, Armonk, NY). Kolmogorov-Smirnov test was used to determine if continuous variables were normally distributed. Descriptive statistics were reported according to data distribution as mean \pm standard deviation (SD), or median and range for continuous variables; the nominal variables were reported as absolute number and percentage (\%). Normally distributed variables were compared between the three groups using the ANOVA test and post-hoc analysis as appropriate. Kruskal-Wallis test by ranks was used to compare nonparametric continuous and ordinal variables with post-hoc analysis if required. 
Qualitative data were compared using the Chi-square test or Fisher's exact test when the expected frequency was less than five. Statistical significance was set at $p<0.05$, after adaptation in case of multiple comparisons with the Bonferroni method. To evaluate whether the $\beta \mathrm{hCG}$ and the EP mass diameter were possible confounders regarding the fertility outcomes, we repeated the analysis between the three groups strafing the study population based on the $\beta$ hCG cutoff value of $1745 \mathrm{mIU} / \mathrm{mL}$, previously reported associated to tubal patency [20], and based on the EP mass diameter of $25 \mathrm{~mm}$.

\section{Results}

In our study period, 228 women were admitted at the University Hospital of Verona with a diagnosis of clinically suspected EP. Twelve of 228 (5.2\%) were excluded from the study population due to extra tubal location of the ectopic pregnancy, in particular $2(0.9 \%)$ had ovarian, $3(1.3 \%)$ cervical, $1(0.4 \%)$ interstitial, $1(0.4 \%)$ subhepatic [21], $1(0.4 \%)$ uterine isthmic and $4(1.7 \%)$ cornual locations. Twelve of $228(5.2 \%)$ women had a history of tubal EP but were excluded from the study because the previous hospitalization was elsewhere and information was not available, or because the previous EP was not during the study period.

One hundred seventy-nine out of 204 (87.7\%) eligible patients responded to the phone call, and 173 out of 179 (96.6\%) women tried to conceive after the index EP episode and agreed to participate. The interval between discharge from the hospital and follow-up ranged from 12 to 80 months. Seventy out of 173 (28.5\%) patients with a diagnosis of tubal EP had initial expectant management, but only $49(70 \%)$ did not receive any treatment following a "waitand-see" approach. Ten (14.3\%) women had plateau BhCG levels and they were subsequently managed with MTX, and another 11 women complained abdominal pain associated with increased levels of $\beta \mathrm{hCG}$ and had surgical management. Overall, 46 out of 173 (26.6\%) received MTX, but in $8(17.4 \%)$ of them, there was the failure of this therapy and were managed surgically. Finally, a total of 86 (49.9\%) women had surgical management. The final success rates were $70 \%$ (49/70), $82.6 \%$ (38/46), and $100 \%$ (86/86) for expectant management, MTX, and surgery, respectively. Based on an intention to treat analysis, the success rate was $70 \%$ (49/70) for the expectant management, 83.3\% (30/36) for the MTX, and 100\% (67/67) for surgery. A flow-chart of patients with ectopic pregnancy was represented in Fig. 1. Patients' characteristics are reported in Table 2 stratified per definitive treatment.

About medical treatment, a single dose of MTX was enough for $33(71.7 \%)$ patients, 4 (8.6\%) patients required two doses, and one patient needed three doses. The eight

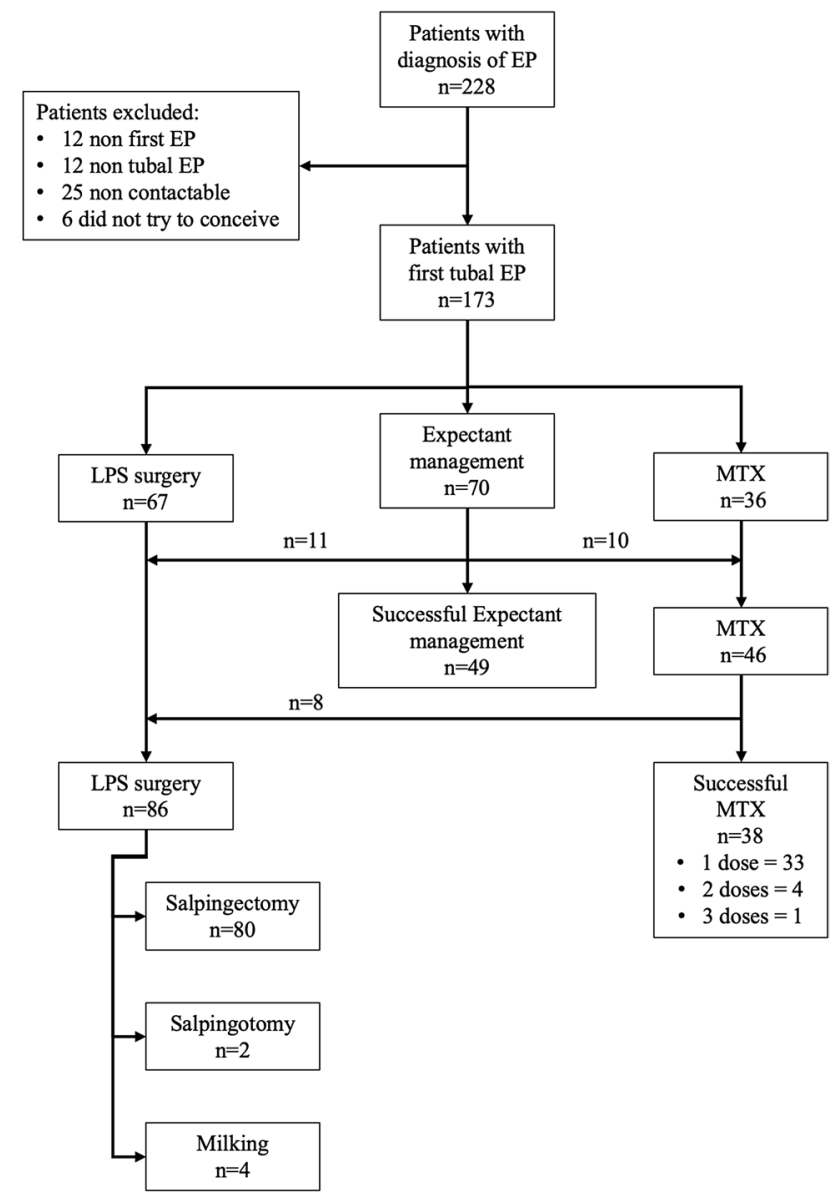

Fig. 1 Flowchart of study population (EP ectopic pregnancy, LPS laparoscopy, $M T X$ methotrexate)

women in the MTX group who failed initial management experienced increasing abdominal pain in the first weeks of follow-up after the first dose of MTX, and they required the surgical treatment. Nine (19.5\%) women treated with MTX reported mild side effects, none needed additional doses, and the therapy was successful for all of them with the spontaneous improvement of all symptoms. Regarding the surgical technique, surgical treatments were salpingectomy for 80 (94.1\%) women, salpingotomy for $2(2.4 \%)$ and tubal milking for $4(4.7 \%)$.

Women were evaluated about their fertility rate and reproductive outcomes after 12-80 months from the index EP. Ten out of 173 (5.8\%) patients referred to assisted reproductive techniques for the attempt of conception after the index EP [six in vitro fertilization and embryo transfer (IVF-ET) and three intracytoplasmic sperm injection (ICSI), 1 intrauterine insemination (IUI)], and seven patients conceived; no significant different distribution regarding the modality of conception was observed among the three groups. Comparing the three treatments, we observed statistically significant differences in terms of cumulative incidence of intrauterine 
Table 2 Characteristics of the study population at the admission stratified per definitive treatment

\begin{tabular}{|c|c|c|c|c|}
\hline & Expectant $(n=49)$ & $\operatorname{MTX}(n=38)$ & Surgery $(n=86)$ & $p$-value \\
\hline \multicolumn{5}{|c|}{ Demographic characteristic } \\
\hline Age (years) & $37.76 \pm 5.68$ & $36.29 \pm 6.07$ & $38.13 \pm 5.26$ & $0.233^{1}$ \\
\hline BMI $\left(\mathrm{kg} / \mathrm{m}^{2}\right)$ & $24.09 \pm 3.14$ & $23.29 \pm 5,04$ & $22.28 \pm 4.11$ & $0.505^{1}$ \\
\hline \multicolumn{5}{|l|}{ Ethnic group } \\
\hline Caucasian & $30(61.2 \%)$ & $27(71.1 \%)$ & $51(59.3 \%)$ & \multirow[t]{5}{*}{$0.708^{4}$} \\
\hline East Europe & $7(14.3 \%)$ & $6(15.8 \%)$ & $13(15.1 \%)$ & \\
\hline Africa & $7(14.3 \%)$ & $3(7.9 \%)$ & $8(9.3 \%)$ & \\
\hline South-East Asia & $3(6.1 \%)$ & $2(5.3 \%)$ & $11(12.8 \%)$ & \\
\hline South America & $2(4.1 \%)$ & 0 & $3(3.5 \%)$ & \\
\hline Smoke $(n)$ & $3(6.1 \%)$ & $3(8.6 \%)$ & $12(15.2 \%)$ & $0.246^{4}$ \\
\hline \multicolumn{5}{|l|}{ Parity } \\
\hline 0 & $23(46.9 \%)$ & $14(36.8 \%)$ & $35(40.6 \%)$ & \multirow[t]{3}{*}{$0.242^{3}$} \\
\hline$>0$ no LB & $11(22.4 \%)$ & $9(23.6 \%)$ & $11(12.7 \%)$ & \\
\hline$>0$ at least one LB & $15(30.6 \%)$ & $15(39.4 \%)$ & $40(46.5 \%)$ & \\
\hline \multicolumn{5}{|c|}{ Clinical data of the day of recovery } \\
\hline \multicolumn{5}{|c|}{ Gestational age } \\
\hline (weeks) & $5.52 \pm 1.81^{\mathrm{a}}$ & $6.34 \pm 1.36^{\mathrm{a}, \mathrm{b}}$ & $6.32 \pm 1.66^{\mathrm{b}}$ & \multirow[t]{2}{*}{$0.037^{2}$} \\
\hline (days) & $38 \pm 12$ & $44 \pm 10$ & $44 \pm 11$ & \\
\hline IUI & 0 & 0 & $2(2.3 \%)$ & $0.359^{4}$ \\
\hline IVF & $1(2.0 \%)$ & $2(5.3 \%)$ & $4(4.7 \%)$ & $0.693^{4}$ \\
\hline \multicolumn{5}{|l|}{ Additional US finding } \\
\hline Blob or bagel sign & $34(69.4 \%)$ & $33(86.8 \%)$ & $60(69.8 \%)$ & \multirow[t]{3}{*}{$0.088^{3}$} \\
\hline Hemoperitoneum & $5(10.2 \%)$ & $2(5.3 \%)$ & $16(18.6 \%)$ & \\
\hline None & $10(20.4 \%)$ & $3(7.9 \%)$ & $10(11.6 \%)$ & \\
\hline Mass size US (mm) & $20.79 \pm 11.75$ & $19.36 \pm 8.88$ & $26.09 \pm 15.27$ & $0.098^{2}$ \\
\hline $\mathrm{Hb}(\mathrm{g} / \mathrm{L})$ & $127.10 \pm 10.45^{\mathrm{a}, \mathrm{b}}$ & $130.01 \pm 10.23^{\mathrm{a}}$ & $121.54 \pm 14.59^{\mathrm{b}}$ & $0.011^{2}$ \\
\hline Leucocytes $\left(* 10^{9}\right)$ & $8.95 \pm 2.64$ & $8.30 \pm 2.00$ & $9.81 \pm 3.82$ & $0.134^{2}$ \\
\hline $\operatorname{PLT}\left(* 10^{9}\right)$ & $252.90 \pm 62.50$ & $264.87 \pm 58.41$ & $256.59 \pm 53.32$ & $0.617^{1}$ \\
\hline ALT (U/L) & $19.4 \pm 7.12$ & $18.85 \pm 10.99$ & $20 \pm 6.96$ & $0.349^{2}$ \\
\hline Creatinine $(\mu \mathrm{mol} / \mathrm{L})$ & $59.9 \pm 8.91$ & $60.58 \pm 8.45$ & $62.33 \pm 8.91$ & $0.627^{1}$ \\
\hline$\beta \mathrm{hCG}(\mathrm{UI} / \mathrm{L})$ & $827.52 \pm 1096.02^{\mathrm{a}}$ & $913.58 \pm 783.09^{\mathrm{a}}$ & $6,165 \cdot 18 \pm 10,243.62^{b}$ & $<0.001^{2}$ \\
\hline \multicolumn{5}{|c|}{ Precedent abdominal surgery } \\
\hline No surgery & $34(69.4 \%)$ & $29(76.3 \%)$ & $58(67.4 \%)$ & \multirow[t]{5}{*}{$0.147^{4}$} \\
\hline LPS & $4(8.2 \%)$ & $4(10.5 \%)$ & $6(7.0 \%)$ & \\
\hline$>1$ LPS & 0 & $1(2.6 \%)$ & $1(1.2 \%)$ & \\
\hline LPT & $7(14.3 \%)$ & $4(10.5 \%)$ & $19(22.1 \%)$ & \\
\hline$>1 \mathrm{LPT}$ & $4(8.2 \%)$ & 0 & $2(2.3 \%)$ & \\
\hline
\end{tabular}

MTX methotrexate, $B M I$ body mass index, $L B$ live birth, IUI intrauterine insemination, $I V F$ in vitro fertilization, $U S$ ultrasound, $H b$ hemoglobin, $P L T$ platelet, $\beta h C G$ beta-human chorionic gonadotropin, $L P S$ laparoscopy, $L P T$ laparotomy

${ }^{1}$ One-way independent ANOVA

${ }^{2}$ Kruskal-Wallis tests

${ }^{3}$ Pearson Chi-square test ( 0 cells with expected less than 5)

${ }^{4}$ Fisher exact test. Each subscript letter (a, b, c) denotes a subset of groups whose column do not differ significantly from each other at the 0.05 level after Bonferroni correction per multiple comparisons
$\mathrm{CP}, \mathrm{LB}$, miscarriage, and time between the index EP and the first intrauterine CP (Table 3). In particular, expectant management was associated with the highest cumulative incidence of $\mathrm{CP}, \mathrm{LB}$, and the shortest time to achieve an intrauterine $\mathrm{CP}$. However, surgery resulted to have the lowest proportion of women who experienced miscarriage. At the post-hoc pairwise analysis, only the expectant management reported statistically significant different reproductive outcomes compared to the surgical approach. Conversely, MTX treatment did not differ significantly from both the other two 
Table 3 Pregnancy outcome after treatment of ectopic pregnancy

\begin{tabular}{cllll}
\hline & Expectant $(n=49)$ & MTX $(n=38)$ & Surgery $(n=86)$ & $p$ \\
\hline CP & $32(65.3 \%)^{\mathrm{a}}$ & $21(55.3 \%)^{\mathrm{a}, \mathrm{b}}$ & $34(39.5 \%)^{\mathrm{b}}$ & $0.012^{1}$ \\
LB & & & $0.035^{2}$ \\
1 & $22(44.8 \%)^{\mathrm{a}}$ & $12(31.5 \%)^{\mathrm{a}, \mathrm{b}}$ & $18(20.9 \%)^{\mathrm{b}}$ & \\
$>1$ & $3(6.1 \%)^{\mathrm{a}}$ & $1(2.6 \%)^{\mathrm{a}}$ & $7(8.1 \%)^{\mathrm{a}}$ & \\
MIS & & & $3(3.5 \%)^{\mathrm{b}}$ & \\
1 & $10(20.4 \%)^{\mathrm{a}}$ & $8(21.1 \%)^{\mathrm{a}}$ & $2(2.3 \%)^{\mathrm{a}}$ & \\
$>1$ & $2(4.1 \%)^{\mathrm{a}}$ & $0^{\mathrm{a}}$ & $7(8.1 \%)$ & $0.220^{2}$ \\
EP & $1(2 \%)$ & $4(10.5 \%)$ & $12.87 \pm 8.89^{\mathrm{b}}$ & $0.041^{3}$ \\
EP_IUP time & $7.92 \pm 6.35^{\mathrm{a}}$ & $13.11 \pm 9.06^{\mathrm{a}, \mathrm{b}}$ & & \\
\hline
\end{tabular}

$C P$ clinical pregnancy (at least one intrauterine pregnancy), $L B$ live birth, $M I S$ miscarriage, $E P$ ectopic pregnancy, IUP intrauterine pregnancy, EP_IUP time Ectopic pregnancy to uterine pregnancy time (months), MTX methotrexate

${ }^{1}$ Pearson Chi-square test

${ }^{2}$ Fisher exact test

${ }^{3}$ Kruskal-Wallis tests. Each subscript letter (a, b, c) denotes a subset of groups whose column do not differ significantly from each other at the 0.05 level, after Bonferroni correction per multiple comparisons managements. No differences were observed regarding EP recurrences. The cumulative incidence of intrauterine $\mathrm{CP}$ after surgery was $37.5 \%(n=30)$ for salpingectomy, $50 \%$ $(n=1)$ for salpingotomy and $75 \%(n=3)$ for tubal milking, with a LB cumulative incidence of $27.5 \%, 0 \%$ and $75 \%$ respectively. No significant differences were observed according to the surgical methods in univariate analysis.

The average size of the EP mass of women who had expectant management was $20.79 \pm 11.75 \mathrm{~mm}$, for women who received MTX was $19.36 \pm 8.88 \mathrm{~mm}$, and for women who underwent surgery was $26.09 \pm 15.27 \mathrm{~mm}$, and the difference was not statistically significant based on the conventional cut-off ( $p=0.098$; Table 2$)$. However, being the EP mass diameter a possible confounder with the mandatory surgical approach in the case of a diameter bigger than $35 \mathrm{~mm}$, we repeated the analysis restricting the study population to patients with an EP mass size of less than $25 \mathrm{~mm}$ (Table 4). The three treatment options reported a statistically significant different cumulative incidence in terms of intrauterine CP and LB. Conversely, miscarriages, recurrent EP, and time to intrauterine $\mathrm{CP}$ were comparable (Table 4). The post-hoc pairwise analysis showed a statistically significant

Table 4 Pregnancy outcome after treatment of ectopic pregnancy considering two cut-offs: ectopic pregnancy (EP) diameter $<25 \mathrm{~mm}$ and betahCG values $<1745 \mathrm{mUI} / \mathrm{mL}$

\begin{tabular}{|c|c|c|c|c|c|c|c|c|}
\hline & \multicolumn{4}{|l|}{ EP mass $<25 \mathrm{~mm}$} & \multicolumn{4}{|c|}{$\beta \mathrm{hCG} \leq 1745 \mathrm{mUI} / \mathrm{mL}$} \\
\hline & Expectant $(n=24)$ & $\operatorname{MTX}(n=24)$ & Surgery $(n=31)$ & $p$ & Expectant $(n=45)$ & $\operatorname{MTX}(n=33)$ & Surgery $(n=26)$ & $p$ \\
\hline $\mathrm{CP}$ & $18(72 \%)^{\mathrm{a}}$ & $12(48 \%)^{\mathrm{a}, \mathrm{b}}$ & $7(21.9 \%)^{\mathrm{b}}$ & $0.001^{1}$ & $27(61.4 \%)$ & $17(48.6 \%)$ & $9(33.3 \%)$ & $0.071^{1}$ \\
\hline \multicolumn{9}{|l|}{ LB } \\
\hline 1 & $11(45.8 \%)^{\mathrm{a}}$ & $7(29.2 \%)^{a, b}$ & $3(9.7 \%)^{b}$ & $0.011^{2}$ & $17(38.6 \%)$ & $10(30.3 \%)$ & $5(19.2 \%)$ & $0.445^{2}$ \\
\hline$>1$ & $3(12.5 \%)^{\mathrm{a}}$ & $1(4.2 \%)^{\mathrm{a}}$ & $2(6.5 \%)^{\mathrm{a}}$ & & $3(6.8 \%)$ & $1(3 \%)$ & $2(7.7 \%)$ & \\
\hline \multicolumn{9}{|l|}{ MIS } \\
\hline 1 & $5(20 \%)$ & $5(20 \%)$ & $2(6.3 \%)$ & $0.139^{2}$ & $9(20.5 \%)$ & $8(22.9 \%)$ & $1(3.7 \%)$ & $0.135^{2}$ \\
\hline$>1$ & $2(8 \%)$ & 0 & 0 & & $2(4.6 \%)$ & 0 & $1(3.7 \%)$ & \\
\hline EP & $1(4 \%)$ & $2(8 \%)$ & $4(12.5 \%)$ & $0.553^{2}$ & $1(2.3 \%)$ & $4(11.4 \%)$ & $2(7.4 \%)$ & $0.252^{2}$ \\
\hline EP_IUPtime & $8.64 \pm 7.51$ & $11.54 \pm 8.65$ & $11.89 \pm 5.54$ & $0.479^{3}$ & $6.94 \pm 5.78^{\mathrm{a}}$ & $13.11 \pm 9.06^{\mathrm{a}, \mathrm{b}}$ & $12.11 \pm 4.01^{\mathrm{b}}$ & $0.032^{3}$ \\
\hline EG & $5.54 \pm 1.67$ & $6.4 \pm 1.61$ & $6.00 \pm 1.33$ & $0.207^{3}$ & $5.52 \pm 1.81$ & $6.18 \pm 2.11$ & $6.33 \pm 1.36$ & $0.145^{3}$ \\
\hline
\end{tabular}

$C P$ clinical pregnancy (at least one intrauterine pregnancy), $L B$ live birth, $M I S$ miscarriage, $E P$ ectopic pregnancy, $I U P$ intrauterine pregnancy, $E P \_I U P$ time ectopic pregnancy to uterine pregnancy time (months), $M T X$ methotrexate, $\beta h C G$ beta-human chorionic gonadotropin

${ }^{1}$ Pearson Chi-square test

${ }^{2}$ Fisher exact test

${ }^{3}$ Kruskal-Wallis tests. Each subscript letter (a, b, c) denotes a subset of groups whose column do not differ significantly from each other at the 0.05 level, after Bonferroni correction per multiple comparisons 
difference in intrauterine $\mathrm{CP}$ and $\mathrm{LB}$ only between expectant and surgical management; in particular, expectant management showed a better reproductive outcome.

In women with expectant management $\beta$ hCG level was $827.52 \pm 1096.02 \mathrm{UI} / \mathrm{L}$, in patients who received MTX injections it was $913.58 \pm 783.08 \mathrm{UI} / \mathrm{L}$, and for women who underwent surgery, it was $6165.18 \pm 10,243.62 \mathrm{UI} / \mathrm{L}$ $(p<0.001$; Table 2$)$. In the post-hoc pairwise analysis, expectant and MTX management were comparable; conversely, both reported $\beta$ hCG values statistically significantly lower than the surgery group. Being the $\beta$ hCG level at diagnosis $(0 \mathrm{~h})$ a possible confounder, we repeated the analysis stratifying women based on the $\beta$ hCG cut-off of $1745 \mathrm{UI} / \mathrm{L}$. In the groups with $\beta$ hCG values lower than $1745 \mathrm{UI} / \mathrm{L}$ (Table 4), we found no statistically significant differences between the three groups, with the only exception for the length of time between the index EP and subsequent intrauterine CP. The expectant management showed the shortest length of time as compared to MTX and surgery, which was statistically significantly different at the pairwise analysis only between expectant and surgical management. For the $\beta \mathrm{hCG}$ level at $0 \mathrm{~h}$ more than $1745 \mathrm{UI} / \mathrm{L}$, only one of the women treated with MTX had a subsequent CP, while the CP cumulative incidence after surgery was $37.9 \%$ and with expectant management was $100 \%(p=0.017)$. Only the expectant management was statistically significantly different as compared to the other two approaches. For all the other outcomes the three groups were comparable.

\section{Discussion}

In the past, EP has been one of the most important causes of maternal morbidity and mortality in the first trimester, accounting for $13 \%$ of the mortality rate [22], but in the last years, due to an earlier diagnosis, its impact has changed allowing a more conservative approach in several situations. In the case of tubal EP smaller than $35 \mathrm{~mm}$ and without embryos heartbeat, if the patient is clinically stable without signs or symptoms of tubal rupture or hemorrhage, three options are available (Table 1). Treatment options include expectant management, medical therapy with MTX, and surgery [23], and the common themes emerging during the discussion with women having clinically suspected EP are the concerns about the treatment effectiveness, the prognosis of future fertility, and the risk of recurrent EP.

Regarding the treatment efficacy, the data are available and comparable to our results, in which we observed $70 \%$ of cases successfully managed with the expectant approach, $82.6 \%$ of cases successfully treated with MTX, and $100 \%$ of success for surgery $[24,25]$. Conversely, the available evidence about the fertility prospects after tubal EP pregnancy treatment is limited. The 2016 RCOG Green-top Guideline stated "there is no difference in the rate of fertility, the risk of future tubal ectopic pregnancy or tubal patency rates between the different management methods" based on lowquality evidence and expert opinion 8/17/2020 5:13:00 p.m.

Our results support this conclusion only partially. Expectant management was associated with the highest cumulative incidence in terms of intrauterine $\mathrm{CP}$ and $\mathrm{LB}$, and to the shortest time interval between the index EP and the intrauterine CP as compared to MTX and surgery, although statistically significant differences were reported only compared to the surgical approach. Therefore, our results suggest that expectant management should be considered the treatment of choice when clinical conditions permit it. These results are further confirmed by the analysis limited to the population with an EP mass diameter lower than $25 \mathrm{~mm}$. Conversely, in the stratified analysis based on the $\beta$ hCG level, only a consistent trend of better reproductive outcomes in women underwent expectant management, without statistically significant differences, was observed; with the only exception for a statistically significant shorter time between the index EP and the subsequent intrauterine CP for the expectant management as compared to the surgical approach.

In general, our results showed a trend of better reproductive outcomes from the surgical approach to the expectant management through the MTX administration, which was confirmed in the stratified analyses for EP diameter and $\beta h C G$ level. Nevertheless, the group that received MTX did not statistically significantly differ from both the expectant management and surgical approach, and the limited sample size of our study population does not allow to exclude a difference between the MTX administration and the other two approaches. However, for what concerns the comparison between MTX and surgery, this is consistent with the results of a meta-analysis that, comparing laparoscopy versus MTX in case of unruptured hemodynamically stable EP, showed that systemic MTX was more cost-effective, with less hospitalization, faster recovery, with no significant differences in subsequent spontaneous conception rate or recurrent ectopic pregnancy [26, 27]. Regarding the concern of leaving a damaged tube that can increase the risk of recurrence after surgery, no statistically significant differences were reported between the three therapeutic options, although a difference in cumulative proportion up to $8.5 \%$ was reported with lower values in the expectant management group as compared to MTX (10\%) and surgery (8.1\%), which were more comparable according to previous studies [28, 29].

About surgery, we did not find any significant difference in reproductive outcomes between the different surgical techniques (salpingectomy, salpingostomy and tubal milking), according to some previous studies [7,30]. Nevertheless, our results are limited by the high proportion of patients underwent salpingectomy (94.1\%), which limits the cases managed with other approaches and the related study 
power to show a difference. The high proportion of patients managed by salpingectomy was because it is the treatment of choice at our Institution; indeed, other studies clearly reported a lower rate of recurrence with no significant difference in future fertility after salpingectomy as compared to salpingotomy $[7,18,19]$.

\section{Strengths and limitations}

The provided evidence about the prognosis of future fertility in these women is limited, mainly because it is affected by confounding factors such as maternal age, previous infertility, history of pelvic/abdominal surgeries or inflammatory diseases, and tubal patency [11]. In our study, age, smoke habit, previous surgeries, BMI, and parity were comparable between the three groups of patients, allowing to exclude a possible effect of these confounders. Moreover, another confounder can be the absence of precise instructions regarding the choice of treatment, which depended on physician preference at the time of diagnosis, in particular in the case of intermediate situations $[8,10]$. Instead, in our study, a preapproved protocol adopted before the study period guided the choice of treatment option in all cases. Finally, the inclusion of only women who actively tried to conceive after the first tubal EP and the long follow-up (12-80 months) strength the study results and limit possible bias present in previous reports [30, 31].

Regardless of strengths, this study has some limitations, which need to be considered for appropriate interpretation of results. Although the investigation of the reproductive outcomes stratified per $\beta \mathrm{hCG}$ values and EP mass diameter allowed to reduce the effect of these possible confounders, the results must be read considering that for high $\beta$ hCG values a comparison is difficult because only surgery was performed according to the protocol, as well as for the increase of the EP diameter. The fact that the chosen treatment was primarily guided by the $\beta$ hCG value and EP mass diameter based on the protocol, which is intrinsically related to the characteristics of the EP, represents the main limitation of the present study. Indeed, we cannot completely exclude that the observed differences between treatments are related to the EP itself instead of the treatment. Nevertheless, assuming that the observed results are related to the EP instead of the treatment, which reflects the underlining characteristics of the EP, this study provides, in any case, evidence able to guide the counseling of patients. In this regard, we cannot confirm that these results are valid if the tubal EP is not managed following the reported protocol (Table 1). These limitations are mainly related to the retrospective study design with the non-randomization of patients to different treatments, which can introduce the possible above-mentioned confounders and limits statistical analysis. Other limitations are the limited sample size, which may explain the absent statistical significance in some observed differences, and the operator-dependence of the US measurement of the EP. Moreover, we cannot completely exclude false positive cases at US, with early intrauterine spontaneous miscarriages confused as EP, although we increased as much as possible the certainty about the diagnosis of tubal EP including in the population only patients with evidence of an EP mass at US.

\section{Conclusion}

Tubal EP is often diagnosed in women who are trying to conceive; therefore, the prognosis of future fertility is one of the main concerns associated with this diagnosis. The results of the present study suggest a progressively better reproductive prognosis from the surgical approach to the expectant management, through the MTX administration, which was confirmed in the stratified analyses for EP diameter and $\beta$ hCG level. Particularly, better reproductive outcomes are reported for expectant management as compared to the surgical approach. Even if the observed fertility outcomes should be more related to the EP mass diameter and the $\beta$ hCG values rather than the adopted treatment, our study provide evidence able to guide the counseling for these patients, particularly if the expectant management is an option. If according to the protocol the expectant management is the treatment of choice, women should be informed that it is effective in more than two-thirds of patients, it is the less invasive option, and it is related to a better prognosis in term of future fertility, with the shortest time to achieve the next intrauterine pregnancy. Conversely, when expectant management is not applicable, medical treatment should be preferred, taking into account women's preferences, and also because it has fewer anesthesia- and surgery-related risks [32]. However, given the unclear differences for subsequent fertility with surgery, the surgical treatment should be considered for women who desire to solve the problem as soon as possible, particularly in the presence of recurrent $\mathrm{EP}$, and for patients whose compliance with immediate follow-up may be doubtful. When clinical presentation and protocol suggest that surgery is the safer and the preferred option, especially in cases of big EP mass with high $\beta$ hCG values, surgery has to be adopted to prevent severe complications such as life-threatening bleedings.

Acknowledgements Thanks for the research contributor: Nikolaous Papadopoulos, MD.

Author contributions All the authors conform the International Committee of Medical Journal Editors (ICMJE) criteria for authorship, contributed to the intellectual content of the study and gave approval for the final version of the article. Material preparation and data collection were performed by $\mathrm{AB}, \mathrm{AR}$, and CQI. Statistical analysis was made by LS and SG. The manuscript was written by SB and SG. Manuscript 
editing and revision were made by ASL, MF, and RR. MF and RR were responsible of project administration and methodology validation. All authors read and approved the final manuscript.

Funding Open access funding provided by Università degli Studi di Verona within the CRUI-CARE Agreement.

\section{Compliance with ethical standards}

Conflict of interest All authors declare that they have no conflict of interest. The authors have no proprietary, financial, professional or other personal interest of any nature in any product, service or company.

Ethical approval The study was in accordance with the ethical standards of the institutional and/or national research committee and with the 1964 Helsinki declaration and its later amendments or comparable ethical standards. The design, analysis, interpretation of data, drafting and revisions conform the Committee on Publication Ethics (COPE) guidelines (https://publicationethics.org/), and the RECORD (reporting of studies conducted using observational routinely-collected health data) statement, available through the EQUATOR (enhancing the quality and transparency of health research) network (https://www.equatornetwork.org). The study was non-advertised, and no remuneration was offered to encourage patients to give consent for collection and analysis of their data. The retrospective study design and development, with anonymized handling of the data, was approved by the Institutional Review Board of the University of Verona (Protocol number 5378, date 29/01/2019).

Informed consent Informed consent was obtained from all individual participants included in the study. Each patient enrolled in this study signed an informed consent for all the procedures and to allow data collection and analysis for research purpose.

Open Access This article is licensed under a Creative Commons Attribution 4.0 International License, which permits use, sharing, adaptation, distribution and reproduction in any medium or format, as long as you give appropriate credit to the original author(s) and the source, provide a link to the Creative Commons licence, and indicate if changes were made. The images or other third party material in this article are included in the article's Creative Commons licence, unless indicated otherwise in a credit line to the material. If material is not included in the article's Creative Commons licence and your intended use is not permitted by statutory regulation or exceeds the permitted use, you will need to obtain permission directly from the copyright holder. To view a copy of this licence, visit http://creativecommons.org/licenses/by/4.0/.

\section{References}

1. Bakken IJ, Skjeldestad FE (2006) Time trends in ectopic pregnancies in a Norwegian county 1970-2004-a populationbased study. Hum Reprod Oxf Engl 21:3132-3136. https://doi. org/10.1093/humrep/del289

2. Tay JI, Moore J, Walker JJ (2000) Ectopic pregnancy. BMJ 320:916-919. https://doi.org/10.1136/bmj.320.7239.916

3. Garzon S, Laganà AS, Pomini P et al (2018) Laparoscopic reversible occlusion of uterine arteries and cornuostomy for advanced interstitial pregnancy. Minim Invasive Ther Allied Technol 4:1-4. https://doi.org/10.1080/13645706.2018.1547764

4. Sagiv R, Debby A, Feit H et al (2012) The optimal cutoff serum level of human chorionic gonadotropin for efficacy of methotrexate treatment in women with extrauterine pregnancy. Int J Gynaecol Obstet 116:101-104. https://doi.org/10.1016/j. ijgo.2011.09.023

5. van Mello NM, Mol F, Verhoeve HR et al (2013) Methotrexate or expectant management in women with an ectopic pregnancy or pregnancy of unknown location and low serum hCG concentrations? A randomized comparison. Hum Reprod Oxf Engl 28:6067. https://doi.org/10.1093/humrep/des373

6. Turan V (2011) Fertility outcomes subsequent to treatment of tubal ectopic pregnancy in younger Turkish women. J Pediatr Adolesc Gynecol 24:251-255. https://doi.org/10.1016/j. jpag.2010.12.007

7. Chen L, Zhu D, Wu Q, Yu Y (2017) Fertility outcomes after laparoscopic salpingectomy or salpingotomy for tubal ectopic pregnancy: a retrospective cohort study of 95 patients. Int J Surg Lond Engl 48:59-63. https://doi.org/10.1016/j.ijsu.2017.09.058

8. Yousefnezhad A, Pirdehghan A, Roshandel Rad M et al (2018) Comparison of the pregnancy outcomes between the medical and surgical treatments in tubal ectopi pregnancy. Int J Reprod Biomed Yazd Iran 16:31-34

9. Jurkovic D, Memtsa M, Sawyer E et al (2017) Single-dose systemic methotrexate vs expectant management for treatment of tubal ectopic pregnancy: a placebo-controlled randomized trial. Ultrasound Obstet Gynecol 49:171-176. https://doi. org/10.1002/uog.17329

10. Demirdag E, Guler I, Abay S et al (2017) The impact of expectant management, systemic methotrexate and surgery on subsequent pregnancy outcomes in tubal ectopic pregnancy. Ir J Med Sci 186:387-392. https://doi.org/10.1007/s11845-016-1419-5

11. Hajenius PJ, Mol F, Mol BWJ et al (2007) Interventions for tubal ectopic pregnancy. Cochrane Database Syst Rev. https:// doi.org/10.1002/14651858.CD000324.pub2

12. Strobelt N, Mariani E, Ferrari L et al (2000) Fertility after ectopic pregnancy. Effects of surgery and expectant management. J Reprod Med 45:803-807

13. Rantala M, Mäkinen J (1997) Tubal patency and fertility outcome after expectant management of ectopic pregnancy. Fertil Steril 68:1043-1046. https://doi.org/10.1016/s0015 -0282(97)00414-7

14. Fernandez H, Lelaidier C, Baton C et al (1991) Return of reproductive performance after expectant management and local treatment for ectopic pregnancy. Hum Reprod Oxf Engl 6:1474-1477. https://doi.org/10.1093/oxfordjournals.humrep.a137292

15. Zohav E, Gemer O, Segal S (1996) Reproductive outcome after expectant management of ectopic pregnancy. Eur J Obstet Gynecol Reprod Biol 66:1-2. https://doi.org/10.1016/03012115(95)02370-4

16. Stovall TG, Ling FW, Gray LA (1991) Single-dose methotrexate for treatment of ectopic pregnancy. Obstet Gynecol 77:754-757

17. Lloyd ME, Carr M, McElhatton P et al (1999) The effects of methotrexate on pregnancy, fertility and lactation. QJM Mon J Assoc Physicians 92:551-563. https://doi.org/10.1093/qjmed/92.10.551

18. Mol F, van Mello NM, Strandell A et al (2014) Salpingotomy versus salpingectomy in women with tubal pregnancy (ESEP study): an open-label, multicentre, randomised controlled trial. Lancet Lond Engl 383:1483-1489. https://doi.org/10.1016/S0140 $-6736(14) 60123-9$

19. Li J, Jiang K, Zhao F (2015) Fertility outcome analysis after surgical management of tubal ectopic pregnancy: a retrospective cohort study. BMJ Open 5:e007339. https://doi.org/10.1136/ bmjopen-2014-007339

20. Seyedoshohadaei F, Mohammadbeigi R, Tahmuri A, Ghaderi E (2016) Frequency and related factors of tubal patency after methotrexate treatment in women with ectopic pregnancy. J Obstet Gynaecol Res 42:286-290. https://doi.org/10.1111/jog.12894 
21. Garzon S, Raffaelli R, Montin U, Ghezzi F (2018) Primary hepatic pregnancy: report of a case treated with laparoscopic approach and review of the literature. Fertil Steril 110:925-931.e1. https:// doi.org/10.1016/j.fertnstert.2018.05.020

22. Nama V, Manyonda I (2009) Tubal ectopic pregnancy: diagnosis and management. Arch Gynecol Obstet 279:443-453. https://doi. org/10.1007/s00404-008-0731-3

23. Sivalingam VN, Duncan WC, Kirk E et al (2011) Diagnosis and management of ectopic pregnancy. J Fam Plann Reprod Health Care 37:231-240. https://doi.org/10.1136/jfprhc-2011-0073

24. Cohen A, Zakar L, Gil Y et al (2014) Methotrexate success rates in progressing ectopic pregnancies: a reappraisal. Am J Obstet Gynecol 211:128.e1-5. https://doi.org/10.1016/j.ajog.2014.03.043

25. Levin I, Tsafrir Z, Sa' ar N, et al (2011) "Watchful waiting" in ectopic pregnancies: a balance between reduced success rates and less methotrexate. Fertil Steril 95:1159-1160. https://doi. org/10.1016/j.fertnstert.2010.10.014

26. Mol F, Mol BW, Ankum WM et al (2008) Current evidence on surgery, systemic methotrexate and expectant management in the treatment of tubal ectopic pregnancy: a systematic review and meta-analysis. Hum Reprod Update 14:309-319. https://doi. org/10.1093/humupd/dmn012

27. Kazandi M, Turan V (2011) Ectopic pregnancy: risk factors and comparison of intervention success rates in tubal ectopic pregnancy. Clin Exp Obstet Gynecol 38:67-70

28. de Bennetot M, Rabischong B, Aublet-Cuvelier B et al (2012) Fertility after tubal ectopic pregnancy: results of a population-based study. Fertil Steril 98(1271-1276):e1-3. https://doi.org/10.1016/j. fertnstert.2012.06.041

29. Bouyer J, Job-Spira N, Pouly JL et al (2000) Fertility following radical, conservative-surgical or medical treatment for tubal pregnancy: a population-based study. BJOG Int J Obstet Gynaecol 107:714-721. https://doi.org/10.1111/j.1471-0528.2000.tb133 30.x

30. Fernandez H, Capmas P, Lucot JP et al (2013) Fertility after ectopic pregnancy: the DEMETER randomized trial. Hum Reprod Oxf Engl 28:1247-1253. https://doi.org/10.1093/humrep/det037

31. Kostrzewa M, Zyla M, Litwinska E et al (2013) Salpingotomy vs salpingectomy - a comparison of women's fertility after surgical treatment of tubal ectopic pregnancy during a 24-month followup study. Ginekol Pol 84:1030-1035. https://doi.org/10.17772/ $\mathrm{gp} / 1675$

32. Nieuwkerk PT, Hajenius PJ, Van der Veen F et al (1998) Systemic methotrexate therapy versus laparoscopic salpingostomy in tubal pregnancy. Part II. Patient preferences for systemic methotrexate. Fertil Steril 70:518-522. https://doi.org/10.1016/s0015 -0282(98)00213-1

Publisher's Note Springer Nature remains neutral with regard to jurisdictional claims in published maps and institutional affiliations. 